\title{
EINE REFLEXION ÜBER DIE ETHNOMATHEMATIK DES MAURERS UND DIE SCHULMATHEMATIK
}

\section{ORIGINAL-ARTIKEL}

SCHWANTES, Vilson ${ }^{1}$

XAVIER, Márcio Pizzete ${ }^{2}$

SCHWANTES, Eloísa Bernardete Finkler ${ }^{3}$

SCHWANTES, Daniel ${ }^{4}$

\footnotetext{
${ }^{1}$ Master in Didaktik der Naturwissenschaften - Mathematik, UNIJUI - RS. Abschluss und Spezialisierung in Naturwissenschaften und Mathematik. Prof. Assistent des CCA - Zentrums für Agrarwissenschaften, Campus von Marschall Cândido Rondon, UNIOESTE, PR - Brasilien.
}

2 Master in Nachhaltiger Entwicklung des ländlichen Raums-UNIOESTE, Mathematikspezialist, Physik-UNIPAR, Personalmanagement und Sonderpädagogik mit Schwerpunkt Mehrfachbehinderung-UNIASSELVI, Abschluss in Mathematik mit Schwerpunkt Physik-UNIPAR.

${ }^{3}$ Spezialisierung im naturwissenschaftlichen Unterricht - Mathematik, Physik und Chemie. UNIOESTE - Staatliche Universität von Western Paraná. Abschluss: Naturwissenschaften und Mathematik. UNIJUI, RS. Professor der staatlichen Schule von Paraná.

${ }^{4}$ Er ist Professor für Pflanzenschutz und menschliche Gesundheit an der Päpstlichen Katholischen Universität von Chile, Abteilung für Pflanzenwissenschaften. Seine interdisziplinäre Position teilen sich die Fakultät für Agronomie und Ingeniería Forestal (FAIF), die Fakultät für Medizin und die Fakultät für Chemie. Forschungsprofessor in der Arbeitsgruppe Boden und Umwelt (GESOMA - UNIOESTE). Master in Agronomie von UNIOESTE, PhD in Agronomie von UNIOESTE (2013-2016) - Sandwich-Periode 
JUNIOR, Affonso Celso Gonçalves ${ }^{5}$

KRACKE, Elisa ${ }^{6}$

JUNIOR, Élio Conradi ${ }^{7}$

SCHWANTES, Vilson. Et al. Eine Reflexion über die Ethnomathematik des Maurers und die Schulmathematik. Revista Científica Multidisciplinar Núcleo do Conhecimento. 04-Jahr, Ed. 07, Vol. 14, S. 87-106. Juli 2019. ISSN: 2448-0959

\section{ZUSAMMENFASSUNG}

Dieser Artikel reflektiert Ethnomathematik, analysiert Interviews mit einer Gruppe von Maurern und Verkäufern von Baumaterial aus den Gemeinden Mercedes und Marechal Céndido Rondon, PR. Es versucht, das mathematische Wissen zu kennen, das von diesen Arbeitern in der Ausübung ihrer Berufe verwendet wird, und ihre mögliche Beziehung zum Unterrichten von Schulmathematik. In den Berichten der

(CAPES-Stipendium) von der Universität Lissabon, am Instituto Superior de Agronomia (ULisboa).

${ }^{5}$ Research Productivity Level $1 \mathrm{C}$ von CNPq im Bereich Umweltwissenschaften mit drei Postdocs, UEM-PR (Brasilien), Universität Santiago de Compostela (Spanien), UFGGO (Brasilien). Derzeit ist er außerordentlicher Professor an der UNIOESTE-PR und Professor und Forscher am Zentrum für Agrarwissenschaften, wo er Chemie lehrt. Dozent im Masterstudiengang Agrarwissenschaften an der UEM. Derzeit ist er Ad-hocBerater bei CNPq, CAPES und Fundação Araucária. Arbeitet als freiwilliger Umweltberater bei MP-SP und CONAMA-DF.

${ }^{6}$ Bachelor in Agronomie - UNIOESTE - Staatliche Universität von Western Paraná Absolvent der Verwaltung - Unip - Universidade Paulista.

${ }^{7}$ Masterstudent in Agronomie (Pflanzenproduktion) an der Staatlichen Universität von West-Paraná (UNIOESTE). Agraringenieur, Absolvent der UNIOESTE (2014-2018), arbeitet als Forscher in der Arbeitsgruppe für Boden und Umwelt (GESOMA UNIOESTE). 
Teilnehmer scheint es, dass dieses Wissen eng mit dem soziokulturellen Umfeld verbunden ist, was auf die Vorstellung hindeutet, dass die Schule ein privilegierter Ort ist, an dem das mathematische Wissen organisiert und systicated erlernt wird. für das Berufsleben notwendig sind. Es ist offensichtlich, dass die Schule Herausforderungen für ihre effektive Konsolidierung zu bewältigen hat, so dass die pädagogische Praxis der Schulbildung Mithänge mit der soziokulturellen Welt des Schülers hat.

Schlagworte: ethnomathematics perspektive, mathematische Ausbildung, mathematisches Wissen, Klassenzimmer Routine.

\section{EINFÜHRUNG}

Der Mathematikunterricht an brasilianischen Schulen war in den letzten Jahrzehnten überwiegend traditionell, ohne größere pädagogische Veränderungen, bis auf wenige Ausnahmen. In dieser Perspektive und Realität wird wahrgenommen, dass von den früheren Säbeln der Schüler wenig verwendet wird, und noch weniger werden Beziehungen der mathematischen Konzepte, die mit dem Leben der Schüler außerhalb des schulischen Umfeldes gelehrt werden, gemacht. In diesem Szenario ist es immer noch üblich, in der Schule Ausdrücke wie zu hören: "Lehrer, wo ich das in meinem Leben verwenden?".

Laut Santos (2016):

Der Mangel an Kontextualisierung in der Lehre der Mathematik kann zur Entmutigung der Disziplin führen, die Wiederauferstehung der traditionellen Methoden, die Mathematik als eine Wissenschaft, die alle Dinge bereit gebracht, als ob es ein fertiges Wissen und über. (p. 5)

In vielen brasilianischen Schulen ist immer noch $\mathrm{zu}$ hören, dass der Mathematikunterricht von der Realität, die Schüler und Bürger erfahren, weit entfernt und dekontextualisiert ist. Für Demo (1996) beginnt der Aufbau des Wissens aus dem Wissen, das jeder Einzelne in seine soziokulturellen Erfahrungen einfließen lässt. Denn der Autor "Alle sprechen, kommunizieren, ein Grundvokabular verwenden, 
Konzepte im gesunden Menschenverstand verwalten, Bezug auf die Realität haben, in die sie eingefügt werden" (S. 32).

Obwohl wir in unseren Schulen immer noch solche Situationen erleben, besteht ein Konsens darüber, dass Schüler, die die Schule betreten, ihr Gepäck mitbringen, auch wenn es nur im gesunden Menschenverstand ist. Dazu gehört das mathematische Wissen, das in der Familie und in den Bereichen der Gesellschaft erzeugt wird, das von den Kindern eingearbeitet wird, und es ist wichtig, dass die Schule es kennt und betrachtet, auf der Grundlage dieser Kenntnisse, des Systate-Wissens. Für Pompey und Monteiro (2001) "lernen die Schüler im täglichen Leben, in ihrer Beziehung zur Welt" (S. 62).

Für Lopes (2014),

"Ideen und Situationen mathematischer Natur sind in den alltäglichen Dingen, in beruflichen Tätigkeiten, in den Praktiken verschiedener Kulturen, in Situationen des Zählens, Messens und Rechnens vorhanden, die leicht als Mathematik erkannt werden." (p.5)

Eine Lehre mit der Produktion von Bedeutungen über den Kontext ist ebenso grundlegend wie die Beziehung zur realen, abstrakten und symbolischen Welt. Ausgehend von der kulturellen Erfahrung eines jeden Schülers, der Bedeutung, die er aus seiner sozialen und kulturellen Erfahrung mitbringt, kann man andere Kulturen, Individuen und Die Gesellschaft voranbringen und verstehen. Wir teilten mit D'ambrosius (1993), als er postulierte, dass es von grundlegender Bedeutung zu verstehen, dass, wenn die formale Schule beginnt, das Kind bereits eine Ethnomathematik hat, die es inm erlaubt, die Interpretationen der systematischen Mathematik der Schule zu stellen.

Diese Perspektive, nach Marques (2001), stellt und bedeutet:

Aufbau neuer Kenntnisse auf der Grundlage von Vorkenntnissen; In der Tat, eine Rekonstruktion von ihnen, im Sinne der Demontage und Erholung in einer neuen Art und Weise. Das Wissen jedes 
Gesprächspartner-Vertrauten, Leser, Autoren, die mit ihren Werken beschworen werden, Themen sozialer Praktiken, denen ich gehört, interviewt, blau-und mein Wissen wird verschmolzen und transformiert, umformuliert. Auf eine ganz besondere Art und Weise konfigurieren meine Vorkenntnisse jetzt andere. Das nennen wir Lernen. Warum nicht forschung? (S. 26).

Wir teilen mit Fiorentini (1995), indem wir hervorheben, dass im ethnomathematikAnsatz der Lehr-Lern-Prozess als Ausgangspunkt die Betonung der Probleme hat, die irgendwie Bedeutung für Kinder haben. In diesem Sinne findet die Beziehung zwischen Schüler und Lehrer im Kontext der Intersubjektivität statt, wo durch Dialog, Interlokation zwischen den Subjekten, Erfahrungsaustausch, beide (re) konstruieren Wissen durch Problematisierung (Mathematisierung) über gesunden Menschenverstand und Wissen Systate, einen neuen Blick auf den Kontext zu konstruieren.

Obwohl es klar ist, dass die Schule an den systematischen mathematischen Kenntnissen arbeiten muss, glauben wir, dass eine Wertschätzung des mathematischen Wissens, das sich aus den kulturellen Beziehungen der Schüler ergibt, das Wachstum positiv und psychologisch machen kann. Das Einkommen der Kinder, im Gegensatz zu einer Initiation durch Techniken und Regeln, um richtige Antworten zu erhalten. Diese Möglichkeit kann mit der Annahme einer Ethnohaltung im Unterricht durch die mathematischen Pädagogen erblickt und konkretisiert werden, denn nach Pompeius und Monteiro (2001) "sucht Ethnomathematik eine Transformation der gegenwärtigen Beziehungen des Menschen mit der Welt" (S. 148 ) .

Um in der heutigen Welt zu handeln, ist es notwendig, eine neue Haltung, ein neues Paradigma, eine theoretische Überprüfung, die als zentrales Ziel die Organisation/Neuformulierung/das Verständnis der theoretischen und praktischen Grundlage hat, die das pädagogische Handeln der Pädagogen durchdringen (SILVA, 2012, S. 174 ). 
Für D'ambrsio (2001) kann Ethnomathematik in der Konstitution des Forschers Lehrer seiner Praxis helfen, er weckt inn auf kontinuierliche Weise zu lernen, ein Lehrer zu sein, nach den aktuellen bildungspolitischen Bedürfnissen, umwandlung des Klassenzimmers in eine Umgebung des Wissensaustauschs, die Rettung historischsozial konstruierten Wissens über die Menschheit.

Schulpädagogische Projekte haben keine Angemessenheit für das neue pädagogische Paradigma, das sich auf Intersubjektivität und Interlocution der Akteure konzentriert. Ein mathematischer Ansatz in dieser Perspektive verwandelt das Klassenzimmer in ein Umfeld des Wissensaustauschs, indem er individuelle Unterschiede bewertet, denn die Erlösung der Neugier erholt das historische Wissen, das der Mensch sozial aufgebaut hat, entlang der Geschichte der Menschheit.

Reflexionen und Diskussionen über den Unterricht dieser Disziplin, besteht auch in der Überprüfung der Erwartungen und sozialen Bedürfnisse der Mathematik in der Schule gelehrt, nach den Worten von Demo (1996, S. 10), ist "aus der geschlagenen reproduktiven Haltung, markiert Vor allem für die wiederholte Klasse zu wiederholen.

Die neuen Trends in der Bildung und Mathematik-Bildung betonen, dass die Kunst des Lehrens nicht nur beinhaltet zu wissen, was zu lehren und über Lehrmethoden zu wissen, sondern vor allem ein Wissen über das Lernen. Wir beziehen uns auf das Lernen, sowohl des Lehrers, über den Prozess selbst und die Ergebnisse seines Lernens, als auch auf das Wissen des Lehrers in Bezug auf das Lernen seiner Schüler. (SILVA, 2012, p.197).

So kann der Unterricht von Mathematik im Klassenzimmer unter Berücksichtigung der Vorkenntnisse und der kulturellen Vielfalt der Schüler eine wichtige Strategie für einen sinnvolleren und weniger traditionellen Unterricht der Schulmathematik sein, was mehr Interesse und Teilnahme der Studierenden am Aufbau mathematischer Kenntnisse. 
Wir glauben, dass eine didaktisch-pädagogische Haltung, die Teil des Wissens, das der Schüler bereits von seiner/ihrer täglichen Erfahrung hat, bietet Pädagogen eine (Re-)Konstruktion des Systate mathematischen Wissens, sowohl in der Wahrnehmung der Existenz von mehreren Mathematik in unserer Mitte, sowie das Streben nach einer Lehre, die soziale Erwartungen betrachtet.

\section{JUSTIING THE PRESENCE OF MATHEMATICS IN THE DAILY ACTIVITY OF THE MASON}

Wenn wir zu verschiedenen Zeiten, die Studiensitzungen mit den Maurern durchführen, versuchen wir, dialogisch mit jedem Profi zu untersuchen, ihre Meinungen über mathematische Fragen von allgemeinem Charakter, die ihre tägliche Maut durchdringen.

Auf die Frage nach dem Einsatz von Mathematik in ihrer Arbeit, waren sich die Maurer einig, dass es bei der Ausübung ihres Berufs von grundlegender Bedeutung sei. Laut Oscar, "vor allem am Anfang eines Werkes, der Klempnerdraht. Mit dem Klempnerdraht machen wir eine Mathematik nicht so kompliziert wie die der Schule und ohne Formeln, ergänzt den Mason. Was wir am meisten verwenden, ist Argumentation, betonend. "

Sérgio kommentiert, dass er Mathematik verwendet "um die Menge der Fliesen zu messen und zu berechnen, Ziegel, manchmal Quadratmeter. Es ist Berechnungen der Ze[multiplicação]iten, me[adição]hr und Splitting. " Alberto sagt auch mit Mathematik "von der Gründung des Baus, um den Platz, durch den Satz des Pythagorean zu nehmen. Auf dem Dach, um den Prozentsatz des Wassers fallen zu lassen. Ich benutze auch Mathematik in dem Bereich Maßnahmen "Enden.

Als wir argumentierten, dass sie eine praktische Mathematik verwendet, Sagte Oscar bald, dass in der Ausübung eines Berufs eine "praktischere und einfachere" Mathematik nicht in den Formeln in der Schule gelehrt verwendet wird. Wir versuchen immer, die Verfahren zu vereinfachen. In einer Konstruktion definieren wir die Art der 
Berechnung, die Sie verwenden werden. Wir verschwenden nicht viel Zeit mit komplizierten Berechnungen, alles wird vereinfacht, argumentiert er. "

Angestiftet, um die Mathematik, die er verwendet besser zu klären und ermöglicht es ihm, Berechnungen vereinfacht $\mathrm{zu}$ machen, Oscar berichtete, dass es "eine Argumentation, die praktisch der Tag-zu-Tag des Erbauers ist und beinhaltet vor allem Maßnahmen. Wir kümmern uns um sie den ganzen Tag und, Sie müssen immer aufmerksam sein. "

Nach Breda, Lima und Guimarées (2011, S. 15):

Ich begann, den Vorschlag der Ethnomathematik als eine Möglichkeit zu betrachten, die Arbeit zu unterscheiden, die der Lehrer in den Schulen entwickelt, das heißt, die conteudistische und bedeutungslose Praxis kann durch einen Lehrer ersetzt werden, der durch ein neues Aussehen orientiert ist, das fördert Die Wertschätzung des soziokulturellen Kontextes des Erziehens, seiner Denkprozesse und seiner Wege des Verstehens, Erklärens und Ausübens seiner Praxis in der heutigen Gesellschaft, eine E[...]inladung, seine pädagogischen Praktiken und seine Auswirkungen zu überdenken oder sogar seine Rolle zu betrachten, Als Dozent, der sich mit unterschiedlichen Sichtweisen im schulischen Kontext auseinandersetzt.

Für die Forschung, Wir halten es für interessant zu fragen, wo und wie sie diese Mathematik gelernt, die die Algorithmen der Operationen verwendet, aber es bringt zugrunde eine Art von Argumentation, die die Lösung der Problemsituationen ermöglicht, denen sie täglich gegenüberstehen. Sérgio sagt, er habe "im Alltag mit den Leuten gelernt, die ich als Maurer gelernt habe. Ich begann als Helfer und lernte, bis ich an diesem Punkt der Autonomkeit ankam. "

Der Mason Oscar sagt in seinem Bericht, er habe "im Hauptkurs die Grundlagen gelernt, aber ein großer Prozentsatz dessen, was wir verwenden, lernt aus der Arbeit selbst. Wenn die Probleme auftauchen, werden Sie lösen und lernen in der täglichen 
Konstruktion selbst. " Nach Pompeu und Monteiro (2001), diese Haltung spiegelt die Annahme der empirischen Konzeption, mit Hilfe der Mathematik, um die unmittelbarsten Problemsituationen zu lösen, die das tägliche Leben der Mason Aktivität durchdringen. Das Wesentliche, so die Autoren, sei es, die praktischen Probleme zu lösen, mit denen die tägliche Maut des Berufs steht.

Auf die Frage, wie er die notwendigen Verfahren erlernt habe, um diesen Beruf auszuüben, berichtete Oscar, dass er "zunächst als Hilfskraft arbeitete und beobachtete, wie es anderen Menschen ging. Ich denke, die Person muss Interesse haben und in der Zeit lernt und perfektioniert, was er tut, andere Wege zu finden, um die Berechnungen zu tun. Du kannst nicht stillstehen, du musst neugierig sein in dem, was du tust, und versuchen, maximale Aufmerksamkeit auf die Perfektionierung zu zahlen und in der Arbeit, die du vollbringt, immer objektiver zu werden. " Nach D'ambrsio (2001) Ein Verhalten, das "das Abenteuer der menschlichen Spezies auf der Suche nach Wissen und bei der Annahme von Verfahren" widerspiegelt (S. 17).

"Ich war immer sehr hingebungsvoll meinem Vater, der Maurer war, ich war sehr noser, also lernte ich viele Dinge von inm. Viele Dinge habe ich auch im Alltag gelernt, aber ohne die Mathematik der Schule wäre es schwierig, meinen Beruf auszuüben, denn es hilft sehr", schließt Alberto. Marques (2000) sagt: "Die Lernprozesse werden unweigerlich in kommunikative und öffentliche Gemeinschaften eingefügt, in denen Die Menschen voneinander und miteinander lernen" (S. 29).

Mathematik muss als soziokulturell-natürliches Produkt eines Volkes interpretiert werden, so Rosa Neto (2002, S. 7 und 19).

Mathematik wurde geschaffen und wurde vom Menschen nach seinen Bedürfnissen entwickelt. (...) Kultur ist eine Form der Anpassung, weil sie eine Art des Handelns auf die Umwelt ist, die mit inr gebaut wurde.

Für Rosa Neto (2002) besteht eine signifikante Beziehung zwischen Mathematik und Kultur, sowohl als Ergebnis unserer Anpassung an unsere Überlebensbedürfnisse im 
Laufe der Zeit, sondern sowohl als empirische, systematische als auch wissenschaftlich.

In Anbetracht der Tatsache, dass die Freimaurer, die an der Forschung teilnehmen, bedenken, dass die Mathematik, von der sie verwenden, in der Praxis des Berufs, ihre beruflichen Bedürfnisse erfüllt und, aufgrund der Tatsache, dass sie in ihrer Grundbildung gelernt wurden, in der täglichen Maut mit anderen Erfahrenere Maurer, wir halten es für relevant zu fragen, ob sie glaubten, dass die Mathematik der Schule könnte die Mathematik, die sie täglich verwenden verbessern.

In den Reden demonstrierten sie den Glauben, dass die Schule ein Ort ist, an dem in organisierter und absichtlicher Weise die Systematisierung des innerhalb oder außerhalb ihrer territorialen Grenzen beschlagnahmten Wissens verarbeitet wird. Oscar erklärte: "Ich glaube, dass die Mathematik der Schule meine Berechnungen verbessern würde, und ich könnte die Dinge einfacher machen, indem ich die Schule die ganze Zeit verlasse. Die Mathematik, die in der Schule gelehrt wird, ist gültig und dient als Ausgangspunkt, um bei dem, was wir tun, zu helfen. Obwohl wir uns in der Regel immer dafür entscheiden, so zu machen, wie Sie im Alltag gelernt haben. " In der Rede des Mason, so Giardinetto (1999), heißt es, "der Zugang zu systematischem mathematischem Wissen war für die Transformation des Alltags lebens unverzichtbar" (S. 7).

Durch diese Verkündigungen wird das Vorhandensein eines Verständnisses deutlich, das von den Reflexionen von Giardinetto (1999) gestützt wird, wobei betont wird, dass in der Tat "das schulische Wissen die Im Alltag vorhandene Denkweise aus Elementen, Keimen Im täglichen Leben präsent und die durch schulische Kenntnisse "(S. 50) eingearbeitet werden. Der Autor sagt: "Die Bildung eines jeden Menschen geht weit über das hinaus, was durch praktisch-utilitaristische Aktivitäten des täglichen Lebens bestimmt wurde" (S. 78).

Für Sergio, "ohne die Mathematik der Schule, auch die vier grundlegenden Operationen würde schwierig werden." Dies zeige laut Giardinetto (1999), dass "das 
Wissen, das jeder Einzelne auf sein tägliches Leben vorbereitet, nicht berücksichtigt wird, um auf die Bedürfnisse seines eigenen täglichen Lebens zu reagieren" (S. 7).

Auf die Frage, wie er Mathematik sehe, die derzeit in der Schule gelehrt wird, postulierte Sergio jedoch, dass es den Menschen helfe und notwendig sei. Er betonte jedoch, dass er Schwierigkeiten hat, mit größerer Tiefe zu opine, "Ich kenne nicht die Mathematik der Schule. Ich habe es nur bis zur vierten Klasse gemacht und meine Kinder sind noch klein", fasst er zusammen.

Laut Oscar ist die heutige Schulmathematik "sehr komplex und sehr kompliziert. Es gibt viele Formeln und im Altag können Sie sie nicht anwenden, so wenig speichern Sie die Formeln im Speicher, um die Berechnungen durchzuführen. Es wäre wichtig, Mathematik bequemer zu unterrichten, mehr in dem, was Sie in Ihrem Täglichen in jedem Sektor verwenden werden. Für meine Bauarbeiten denke ich, dass diese Schulformeln wenig mit dem zu tun haben, was ich jeden Tag brauche. "

Über die Bedeutung der Beziehung des mathematischen Inhalts mit einer realen Situation, Alberto kommentiert: "Es ist wahr, wie im Fall der Pythagorean Satz, vor Jahren, um den Platz des Hauses zu entfernen, benutzten wir das eigene Quadrat und war ausgerichtet, bis Holen Sie sich das richtige Filmmaterial. Jetzt, vor kurzem entstanden für uns die $3 \mathrm{~m} ; 4 \mathrm{~m}$ und $5 \mathrm{~m}$, die auch das Haus auf dem Platz verlässt. Ich habe in der Schule gelernt, dass dies der Satz des Pythagoreans ist, ich meine, indem ich die 3m-Maßnahme mache; 4m, die Diagonale mit $5 \mathrm{~m}$ setzt alles in den Platz ".

Wenn auf die Mathematik an der Schule gelehrt Alberto sagt, dass "sie ist sehr theoretisch, sollte mehr den praktischen Teil bringen. Die Arbeit der Praxis, nicht nur die Theorie, dies wäre sehr wichtig für das Kind, hilft in der intellektuellen Entwicklung. Das Kind, das das Theoretische und praktische hat, wird in das klügste Berufsfeld eintreten", schließt der Maurer.

Und in seiner Rede berichtet Oscar immer noch, dass wir bei der Ausübung seines Berufs "viele Male bestimmte Berechnungen durch andere Formeln lösen können, einfacher als die, die von der Schule gelehrt werden". Sicherlich sollten wir uns in 
diesen Fällen so verhalten, wie sie Lins und Gimenez (1997) vorschlagen, also "von dem, was am vertrautesten ist" (S. 10). Mit dieser didaktischen Haltung, so die Autoren, würde das, was in der Schule gelernt würde, nicht "nur für die Schule" dienen (S. 17), weil sie eine direkte Beziehung zu den Bereichen der täglichen Tätigkeit haben würden.

Indem er dazu angeregt wird, darüber zu sprechen, wie man ihnen die Mathematik der Schule beibringen möchte, sagt Alberto bereitwillig: "Mit praktischen Beispielen, die Bewerbungen in verschiedenen Berufen suchen: Handel, Maurer, Schreiner, unter anderem. Praktische Probleme, Mathematik nicht nur durch Übungsauflösung zu unterrichten. Diese mathematischen Probleme können den Studenten dazu veranlassen, die Theorie zu assimilieren und alles besser zu verstehen, sonst ist es bedeutungslos. Ich lernte den Satz des Pythagoreans in der Schule, aber der Lehrer zeigte nicht, dass es im Alltag dienen, vervollständigt den Maurer.

Die Aussprachen der Maurer zeigen nach der Perspektive lins (1994) in seinen Studien über das theoretische Modell semantischer Felder, "dass derselbe Text, der mit unterschiedlichen Rechtfertigungen gesprochen wird, ein anderes Wissen darstellt[8]" (S. 29).

Um die Reflexion, die wir vorgeschlagen haben, zu endestehen, lassen wir sie sich wohl, um etwas mehr über Mathematik in der Schule und die Mathematik, die sie in der Ausübung ihres Berufs verwenden, zu sagen. Oscar kehrte zurück, um in seiner Rede die Bedeutung der Schulmathematik zu priorisieren: "Die Grundlagen, die ich in der Schule gelernt habe und heute in meinem Beruf benutze. Die Mathematik der Bücher, die Sie heute verwenden, ist fortgeschrittener und ich benutze vor allem nicht viel in meiner Arbeit, aber es kann in anderen Bereichen und in anderen Sektoren verwendet werden.

Die Mathematik, die ich in der Schule gelernt habe, und meine Eltern haben gelernt, viel Gedächtnis zu verwenden. Heute verwenden wir die Formeln und den Rechner. Ich benutze sogar keinen Rechner, ich ziehe es vor, mit Speicher zu arbeiten, obwohl 
der Rechner viel praktischer ist. In meiner Arbeit muss ich in vielen Situationen Dinge mit der Fähigkeit des Gedächtnisses definieren, Dinge erfassen, fertigstellen. "

Nach Alberto die Mathematik der Schule "hilft viel. Es war sehr gut für mich zu studieren. Die Erfahrung, die ich mit meinem Vater gemacht habe, war sehr wichtig, aber ohne Schule nicht. Alles im Schrei zu lösen funktioniert nicht. Ich denke, die Mathematik der Schule hat eine sehr große Bedeutung in meinem Beruf. Die Mathematik der Schule half mir, viele Fehler zu vermeiden, die ich zuvor begangen hatte. Alle Arbeit im Bau getan wird mit einer Art von Mathematik durchgeführt. "

Laut D'ambrsio (2001) zeigt das Zeugnis des Mason, dass "Sie zumindest mit dem mathematischen Pädagogen verwechselt werden, der nicht erkennt, dass es viel mehr in seiner Mission als Pädagoge als Lehre, um Kontinuierlichen zu machen oder Gleichungen und Probleme absolut zu lösen Künstlich, auch wenn es oft den Anschein hat, sich auf reale Tatsachen zu beziehen "(S. 46).

In Bezug auf die Bedeutung, die der Mathematik von der Schule gelehrt, Oscar war emphatisch, sagte, dass "wir können nicht ausschließen, die Mathematik der Schule, weil es lehrt die Prinzipien der Berechnungen und ohne sie wird alles kompliziert. Es stellt sich heraus, dass die Mathematik der Schule wird kompliziert wegen vieler Formeln und dies, für den Maurer, bringt eine gewisse Unzufriedenheit unter den Schülern selbst.

Für Rolim,

Über den historisch-kulturellen Kontext im Prozess des Mathematikunterrichts nachzudenken, ist zu bedenken, dass "Tun" mehr beinhaltet als Regeln und Techniken; ist anzuerkennen, dass die Mathematik als Wissenschaft selbst ein Gebäude der Menschheit ist (ROLIM, 2010, S. 43).

Mit Desinteresse entkommt die Studentin der Mathematik, ist erschrocken und dies muss eine Beziehung mit der Art und Weise haben, wie sie gelehrt wird. Ich glaube nicht, dass ich viel über dieses Thema sprechen sollte, weil ich nicht zu viel zur Schule 
gegangen bin. Was ich gesagt habe, ist wegen dem, was ich im Schulleben meiner Kinder verfolgt habe, und versucht, ihnen bei einigen Gelegenheiten zu helfen, und ich kann sagen, dass ich nicht in der Lage war, die meisten Probleme und Probleme zu lösen, weil es schwierig ist, die Formeln zu verstehen, die heute in der Schule verwendet werden."

Die anfängliche Bestätigung des Maurers gibt laut Giardinetto (1999) die Idee, dass "es notwendig ist zu verstehen, dass Wissen im Alltag ein fragmentiertes Wissen ist, das sich nach einer konzeptuellen Logik manifestiert, die den Anforderungen des täglichen Lebens entspricht. Dies ist eine konzeptionelle Logik, die den praktischen utilitaristischen Zielen entspricht und effektiv auf die Notwendigkeiten des täglichen Lebens reagiert"(S. 6).

Angesichts von Oscars Rede, fragen Sie ihn, ob er dachte, dass oft der Unterricht der Mathematik war weit von einer realen Situation, zu dem, was er vereinbart zu sagen: "Genau, wenn ich auf die Mathematik, die ich in meiner Arbeit und die, die in den Büchern ist, 99\% Benutze ich nicht im Alltag.

In diesem Sinne denke ich, dass es notwendig ist, über dieses Thema nachzudenken und in der Zukunft zu überprüfen, wie man moderne Mathematik mehr angewendet. " Laut Giardinetto (1999) geht es darum, die "Schultätigkeit als Vermittler dieser Beziehung zu verstehen, um die unverzichtbare Rolle der Schule für die Bildung des Individuums zu retten, die oberflächlichen und unmittelbaren Interpretationen zu überwinden, die Sie überschätzen das tägliche Wissen auf Kosten der Aneignung von Schulwissen "(S. 12).

Laut Marques (2000) "die Bildung in ihren verschiedenen Instanzen, insbesondere in der Schule, neigt dazu, das Lernen im Hinblick auf die Produktion des Individuums, das von der aktuellen Kultur modelliert wird, zu standardisieren". Angesichts dieser Beobachtung des Autors haben wir versucht zu zeigen, dass das mathematische Wissen existiert und von den Menschen der Gemeinschaft verwendet wird, aber nicht in einer einzigartigen und standardisierten Weise. In diesem intersubjektiven Szenario glauben wir, dass nur ein Ethno-Ansatz dem Unterricht der Schulmathematik die 
diskursive Debatte über das im Umlauf befindliche Wissen in den verschiedenen menschlichen Aktivitäten bieten wird, indem beides rekonstruiert wird (S. 34).

Die Mathematik kann jedoch Wissen und Verständnis stutze, die nicht in die institutionalisierten Strukturen durch Lehrpläne und Forschungsprogramme passen. In diesem Sinne wäre es möglich, die Mathematik, die im Alltag vieler Berufe vorhanden ist, hervorzuheben. Es ist ein integraler Bestandteil der Technologie, Design und Entscheidungsfindung, ist in Tabellen, Diagrammen und Grafiken. Einfach in einer Zeitung stöbern, um viel Mathematik zu finden (SKOVSMOSE, 2014, S. 13-14).

Die pädagogische Vermittlung, nach der Perspektive vygotskys (2001) monopolisiert den Diskurs des Klassenzimmers nicht, verwandelt inn jedoch in eine argumentative Lerngemeinschaft, in der die Rolle beider im Prozess der (Wieder-)Konstitution der Lernenrelevant ist. In dieser Perspektive würden die Teilnahme der Schüler am Klassenzimmer und die Beteiligung der Sektoren, aus denen die Schulgemeinschaft besteht, auf der breiten Liste stehen und ständig ein Klima kognitiver Debatten zwischen ihnen schaffen, das das Lernen beider rekonstruiert und erneuert.

In den Besuchen, die wir in drei Geschäften gemacht, die Baumaterial resend, es zog unsere Aufmerksamkeit, sowohl der gute Service der Mitarbeiter, was die Manifestationen der Sorge der Manager, als der Mangel an Schulungen für die Maurer auf Generalmode-Konstruktion und Keramikbeschichtung im Besonderen.

In einem der besuchten Läden, als das zentrale Thema der Untersuchung die Ohren der Firmenchefs erreichte, wurden wir zuerst dem Bauingenieur und inm dem Eigentümermanager vorgestellt. Im Dialog hören wir von ihnen viele Ängste und Sorgen. Alle Bedenken waren auf die dekontextualisierte Behandlung der Schulmathematik gerichtet, sowie das Fehlen ihrer Dominanz durch die Maurer im Allgemeinen, in der Ausübung ihrer Berufe. 
In ihren Reden berichten sie von routinemäßigen Kundenbeschwerden im Zusammenhang mit der Ungenauigkeit der Keramiksiedlung, insbesondere in Fällen, in denen Kunden dies wünschen, indem sie die Keramik diagonal-45 o mit der Wand verlegen. Nach den Zeugenaussagen gibt es in dieser Region des Bundesstaates Parané kaum eine Wand oder einen Boden im Klempner und Platz, was auch jede keramische Siedlung gefährdet.

Sie würdigen einen Teil dieser Verantwortung auf die Schulmathematik, die nach innen, hat nur dazu gedient, die Routineaktivitäten der Schule selbst zu befriedigen. In seinen Reden scheint es, nach dem Verständnis von Pompeius und Monteiro (2001), dass "wir einen Moment leben, in dem die Fragmentierung des Wissens das Verständnis der Realität einschränkt, die Sch[porque]ulorganisation an der Aufrechterhaltung eines analytischen, kartesischen Lehrplansystems festhält" ( S. 19). Es ist daher von grundlegender Bedeutung, dass der Unterricht der Schulmathematik die Inhalte kontextualisiert und sie mit den Aktivitäten in Beziehung setzt, die von den Bürgern in den verschiedenen Bereichen der menschlichen Tätigkeit entwickelt wurden.

In seinem kritischen Argument, in Bezug auf die Art und Weise, wie die Schule derzeit Mathematik unterrichtet, berichtet der Unternehmer, dass vor kurzem ein Lord - PitDrill, kam mehrmals in seinem Geschäft fragen, wie viele Kubikmeter würde den Brunnen haben, die gerade hatte bohren. "Das letzte Mal waren Sie hier", sagt der Unternehmer, "er kommentierte mit mir, dass er 20 Jahre lang Sägewerksbesitzer im Bundesstaat Santa Catarina gewesen sei. Ich fragte inn dann, "kommentiert der Besitzer des Ladens", wenn er wusste, wie man Cubicar Holz. Zu meiner Überraschung antwortete der Boxenstanzer prompt ":" Sicher, ich kaufte und verkaufte Holz, musste wissen, wie viele Kubikmeter hatte jede Tora. "

"Ich habe den Grubenbohrer zu folgendem gefragt": "Wenn wir e[relata o empresário]inen runden Baumstamm in diesen Brunnen steck[circular]en, wüssten Sie dann, wie man die Kubikmeter des Brunnens berechnet?" "Ja, es ist einfach", antwortete der Mann, und laut dem Unternehmer begann sein Gesicht in diesem 
Moment "rot" zu werden, weil er es nun geschafft habe, die Verbindung zwischen den beiden Situationen herzustellen.

Während unserer Prosa, sowohl der Unternehmer und der Bauingenieur akzentuierte den Mangel an Interaktion zwischen der Mathematik der wissenschaftlichen Gemeinschaft und der Mathematik in den verschiedenen menschlichen Aktivitäten praktiziert, aufgrund der Mathematik-Klassen beschränkt auf Formaler Geltungsbereich.

Solche Situationen zeigen, wie die Schulmathematik von den realen Situationen, die die Bürger täglich erleben, getrennt ist. Die Bürger wissen jedoch, dass sie dieses Wissen brauchen, aber sie haben Schwierigkeiten, Verbindungen herzustellen. Diese Beobachtungen zeigen im Verständnis von Pompeius und Monteiro (2001), dass "die Lehrer von heute eine große Herausforderung haben: in einem fragmentierten Prozess erzogen, müssen sie die Grenzen überwinden, die diese Formation ihnen auferlegt, und die Grenzen der Inhalte, die teilweise und a-historisch betrachtet werden "(S. 15).

Es geht nicht darum, formales oder informelles Wissen zu überbewerten. Die Gestellte Frage zielt darauf ab, über die effektiven Möglichkeiten der Verbindung von Mathematik in Schulen mit mathematischen Praktiken in menschlichen Aktivitäten praktiziert zu reflektieren. Dabei suchen wir reflexartig nach konvergenten Punkten, denn nach Pompeiund und Monteiro (2001) "wenn Bildung grundsätzlich in einer Beziehung stattfindet" mit "dem anderen, zur Welt, kann weder der andere noch die Welt fragmentiert gesehen werden" (S. 24).

All diese Perspektiven verdeutlichen, wie wichtig es im Mathematikunterricht, beim Aufbau von Wissensbindungen der Gemeinschaft mit dem Wissen der Schule und umgekehrt ist. Diese Dynamik bietet eine (Re-)Konstruktion beider Kenntnisse. Theoretisch ermöglicht die Schule den Schülern, bei der Arbeit in dieser Perspektive besser und systematischzu verstehen, was sie tun und warum sie mathematisch vorgehen. 
Nach dem Verständnis von Pompeius und Monteiro (2001) beginnt "ein sinnvoller Bildungsprozess mit dem Zusammenspiel von Schule und Gemeinschaft" (S. 55), dessen Beziehungen die Rolle der Schule als privilegierter Ort für den Erfahrungsaustausch mit Das wissen, was im Alltag zirkuliert, besser verstehen.

Die Annahme einer pädagogischen Praxis, die die Technik und die Perspektive der Verknüpfung der Lebenswelt mit der Schulwelt verlässt, kann zur Minimierung des Prozesses der Ausgrenzung eines reflektierenden kritischen mathematischen Wissens über die Realität beitragen. Laut D'ambrsio (1996) bedeutet dies die Annahme einer sozio-interagierenden Haltung, die ein neues pädagogisches Paradigma anstrebt, das das bereits abgenutzte Lehren-Lernen ersetzt, basierend auf dem veralteten Verhältnis von Ursache-Effekt.

Wir verstehen und teilen mit Marques (2000), dass kein Wissen in seinem Handlungsbereich geschlossen werden kann. Es muss sozialisiert werden, (re) sich vollständig in und durch die kommunikative Aktion unter den Akteuren Themen etabliert konstruieren. Mit und durch den Gebrauch der Sprache kann man in einer interaktiv-dialogischen Perspektive die (Re-)Konstruktion beider Lernene liefern, indem man die Fächer singulär macht, nicht der Unterwerfung unterworfen.

Dass aus dieser Reflexion die pädagogische Praxis im Klassenzimmer auf die Entwicklung der vollen Bürgerschaft der Schüler umgestaltet werden kann. Das, nach Pinheiro und Rosa (2016), die:

[...] Mathematiklehrer tauchen in die kulturelle Dynamik der Schüler ein und nutzen Lehr- und Lernstrategien, die die kulturelle Dimension im Klassenzimmer schätzen, so dass eine inklusive mathematische Bildung entwickelt werden kann, die effektiv Beitrag zur gesellschaftlichen Transformation (S. 79).

Eine Lehre mit dem Schwerpunkt der Ethnomathematik, glauben wir, den Schülern, Bedingungen, um in die Realität durch das wissen in der Geschichte produziert stören, den Schüler in den Mittelpunkt des Bildungsprozesses, als ein aktives Subjekt in der 
(Re-)Konstruktion seiner wissen. Das zeigten die Reden der Maurer, denn aus ihrer Arbeitstätigkeit erschaffen sie Prozesse ihrer eigenen Gedanken und Strategien, mit Inspiration für ihr Vorwissen und Lernen, das sie im Laufe ihrer Lebensgeschichte erworben haben.

\section{ZIELE}

4.1 Von der Reflexion an den Mathematik-Pädagogen zu beitragen, eine investigative kritische Haltung in Bezug auf den Unterricht der Mathematik zu nehmen, seine Lehrtätigkeit durch die Herstellung von Bindungen zwischen täglicher Mathematik und Mathematik zu initiieren schule 4.2 Beitrag zur Konstitution des forschern Lehrers seiner Praxis, ermuntern ihn/sie, kontinuierlich zu lernen, ein Lehrer zu sein, entsprechend den aktuellen pädagogischen Bedürfnissen, das Klassenzimmer in ein Umfeld des Wissensaustauschs, der Rettung Historisches Wissen- sozial von der Menschheit aufgebaut; 4.3 Vertiefen Sie das Verständnis der Ethnomathematik, indem Sie versuchen, die Rolle der verschiedenen kulturellen Kontexte in der mathematischen Bildung zu verstehen, die durch die Annäherung zwischen Mathematik und Realität ermöglicht wird.

\section{METHODIK}

Wir entwickeln die Arbeit durch verschiedene Momente, nämlich: theoretische Studie zum Thema Ethnomathematik, Gespräche mit Maurern und Verkäufern von Baustoffläden. Wir untersuchen dialogisch mit jedem Fachmann über das mathematische Wissen, das in seiner Tätigkeit verwendet wird. Wir versuchen, pädagogisch über mögliche Zusammenhänge zwischen Mathematik und Realität in der Möglichkeit der Herstellung von Bindungen zwischen dem mathematischen Wissen von Maurern und Schulmathematik zu reflektieren.

\section{ERWARTETER BEITRAG}

Es ist zu hoffen, dass der Unterricht Mathematik im Klassenzimmer durch den ethnograp ${ }^{[9]}$ hischen Ansatz ermöglicht Lehrer und Schüler über die kulturelle Vielfalt 
der Mathematik zu lernen. Dass die Ergebnisse dieser Forschung bei der Entwicklung einer Methodik helfen, die zur Lehre der grundlegenden Mathematik beiträgt, mit Ethnomathematik als Unterstützung für dieses Verständnis.

Mit den Worten von RAM one,

[...] Mathematik unterricht in dieser Konzeption wird es dem Schüler ermöglichen, die Inder-Konzepte mit ihrer täglichen Erfahrung zu verknüpfen, entsprechend ihrem natürlichen, sozialen und kulturellen Umfeld. Es geht nicht darum, die akademische Mathematik abzulehnen, sondern Werte zu berücksichtigen, die in Gruppenerfahrungen erfahren sind, wenn man die historisch-kulturellen Bindungen betrachtet (CARNEIRO, 2012, S. 3).

Durch den erstellten Text, das Ergebnis von Lesungen und Studiensitzungen, zielt es darauf ab, pädagogisch, die Union der Theorie und Praxis für die (Re-)Konstruktion von systematisiertem Wissen. Es ist auch zu hoffen, dass die Reflexionen, die sich aus der Lektüre des Textes ergeben, einen neuen Blick auf die Realität und die mathematische Ausbildung ermöglichen, aus der Perspektive der Produktion von Wissen aus der täglichen Praxis und der Herstellung möglicher Verbindungen zwischen Mathematik und Realität.

\section{BIBLIOGRAPHISCHE HINWEISE}

BREDA, Adriana LIMA, Valderez Marina do Rosário e GUIMARÃES, Gleny Terezinha Duro. A utilização da Etnomatemática nos cursos de formação continuada de professores: implicações das relações de poder saber na produção de subjetividades. Curitiba. Novembro de 2011. Disponível em: http://educere.bruc.com.br/CD2011/pdf/4668_2898.pdf. Acessado em: 20/08/2017.

CARNEIRO, K. T. A. Cultura Surda na aprendizagem matemática da sala de recurso do Instituto Felipe Smaldone: uma abordagem etnomatemática. Anais do 4ํㅡㄹ Congresso Brasileiro de Etnomatemática. Belém, PA: ICEm4, 2012. 
D'AMBROSIO, Ubiratan. Etnomatemática - elo entre as tradições e a modernidade. Coleção Tendências em Educação Matemática, 1. Belo Horizonte: Autêntica, 2001, $112 p$.

Etnomatemática: Arte ou técnica de explicar e conhecer. Editora Ática, Série Fundamentos, 2. edição, São Paulo, 1993.

Educação Matemática: da teoria à prática. Editora Papirus (Coleção Perspectivas em Educação Matemática), Campinas - São Paulo, 1996.

DEMO, Pedro. Pesquisa e construção de conhecimento: Metodologia científica no caminho de Habermas. 3.ed. Rio de Janeiro: Tempo Brasileiro, 1996. 125p.

FIORENTINI, Dario. Alguns modos de ver e conceber o ensino da matemática no Brasil. In. Zetetiké. Campinas, São Paulo, ano 3, n.4,p.1-37, 1995.

GIARDINETTO, José Roberto Boettger. Matemática escolar e matemática da vida cotidiana. Coleção polêmicas do nosso tempo, autores associados, Campinas - São Paulo, 1999, 128p.

LOPES, Antônio José. Os saberes das crianças como ponto de partida para o trabalho pedagógico. In: BRASIL. Secretaria de Educação Básica. Diretoria de Apoio à Gestão Educacional. Pacto Nacional pela Alfabetização na Idade Certa: Apresentação / Ministério da Educação, Secretaria de Educação Básica, Diretoria de Apoio à Gestão Educacional. - Brasília: MEC, SEB, 2014.

LINS, R. C.; GIMENEZ, J. Perspectivas em Aritmética e Álgebra para o Século XXI. Série Perspectivas em Educação Matemática. SBEM. Campinas: Papirus Editora, 1997, 176p.

LINS, Rômulo Campos. 0 modelo teórico dos campos semânticos: uma análise epistemológica da álgebra e do pensamento algébrico. In: Dynamis, v.1, n.7, p. 29-39, abr/jun, Blumenau, 1994. 
MARQUES, Mario Osorio. A aprendizagem na mediação social do aprendido e da docência. ljuí, Editora UNIJUÍ, 2. edição, 2000, 144 p.

Escrever é Preciso: o princípio da pesquisa. ljuí, Editora UNIJUÍ, Coleção educação, 4. edição, 2001, 163p.

MONTEIRO, A.; POMBEU, G. Jr. A matemática e os temas transversais. Editora Moderna, São Paulo, 2001, 160p.

PINHEIRO, R. C.; ROSA, M. Uma perspectiva etnomatemática para o processo de ensino e aprendizagem de alunos Surdos. RPEM, v. 5, n. 9, p. 56-83, 2016.

ROSA NETO, R. Didática da matemática, São Paulo: Ática, 2002.

ROLIM, Carmem Lucia Artioli. Fórmulas de Silêncio: metodologias no processo de ensino da Matemática. In: SANTOS, Jocyléia Santana. ZAMBONI, Ernesta. Potencialidades Investigativas da Educação. Goiânia: Ed. da PUC Goiás, 2010.

SANTOS, Lijecson Souza dos. Contextualização Matemática Em Situação De Ensino E Aprendizagem No Eja. Disponível em: https://editorarealize.com.br/revistas/cintedi/trabalhos/TRABALHO_EV060_MD1_SA 18_ID 492_23102016194610.pdf. 2016. Acesso em 11/11/2017, Hora: 22hrs e 38 min.

SILVA. Adelmo Carvalho da. et. al. Paradigmas Educacionais: Contribuições para - Ensino de Matemática. In. SILVA, Adelmo Carvalho. et al, Ensinar matemática: Formação, investigação e práticas docentes. Cuiabá, MT: DUFMT, 2012.

SKOVSMOSE, Ole. Um convite à educação matemática crítica. Tradução de Orlando de Andrade Figueiredo. Campinas: Papirus, 2014.

VYGOTSKY, Lev Seminovich. A construção do pensamento e da linguagem. Tradução: Paulo Bezerra, São Paulo, Martins Fontes, 2001, 496p.

8. Wissen wird in dieser Perspektive als Glaubensbejahung verstanden, zusammen mit einer Rechtfertigung für Glaubensbejahung. 
9. In dieser Arbeit verwendet, um die Untersuchung der Konzeptionen, Traditionen und mathematischen Praktiken einer sozialen Gruppe und die p[pedreiros]ädagogische Arbeit, die in der Perspektive entwickelt werden kann, dass die Gruppe interpretiert und kodifiziert inr Wissen; Erwerben Sie das Wissen, das von der akademischen Mathematik produziert wird, indem Sie, wenn sie mit kontextualisierten Situationen konfrontiert werden, diejenige, die besser geeignet scheint.

Eingereicht: Juni 2019.

Genehmigt: Juli 2019. 